(2) Open Access Full Text Article

METHODOLOGY

\title{
Impact of the Look-Back Period on Identifying Recurrent Myocardial Infarctions in the Danish National Patient Registry
}

\author{
Søren Korsgaard (iD) ${ }^{1,2}$ \\ Christian Fynbo \\ Christiansen (D) ${ }^{1,2}$ \\ Morten Schmidt (D) ${ }^{1-3}$ \\ Henrik Toft Sørensen (1D ${ }^{1,2}$ \\ 'Department of Clinical Epidemiology, \\ Aarhus University Hospital, Aarhus, \\ Denmark; ${ }^{2}$ Department of Clinical \\ Medicine, Aarhus University, Aarhus, \\ Denmark; ${ }^{3}$ Department of Cardiology, \\ Aarhus University Hospital, Aarhus, \\ Denmark
}

Purpose: Studies examining myocardial infarction (MI) often seek to include only incident MIs by excluding recurrent MIs. When based on historical data, identification of previous MI depends on the length of the look-back period. However, international registries often cover a short time period, consequently containing left-censored data, making it impossible to determine if a first MI in a period is truly an incident MI. We evaluated whether the proportion of MIs identified as recurrent MIs depends on the look-back period, and how including recurrent MIs in a planned incident MI cohort impacts survival estimates.

Patients and Methods: We used the Danish National Patient Registry, covering all Danish hospitals since 1977 to identify first MIs during 2010-2016 (index events). The hospital registry history preceding the index event was then searched for previous MIs. We plotted the proportion of index events identified as recurrent MIs as a function of the look-back period. Moreover, we calculated 5-year all-cause mortality and confidence intervals (CIs) using the 1-Kaplan-Meier method for five cohorts based on the index events and defined by look-back periods of $0,5,10,20$, and up to 39 years.

Results: Among 63,885 index events, 3.4\% were identified as recurrent MIs with 5 years of look-back, $7.9 \%$ with 10 years, $14 \%$ with 24 years, and $15 \%$ with up to 39 years. All-cause mortality risk was $36 \%$ (95\% CI: $36-37 \%$ ) with 0 years of look-back, $35 \%$ (95\% CI: $35-$ $36 \%$ ) with 5 years, 35\% (95\% CI: 35-36\%) with 10 years, 34\% (95\% CI: 34-35\%) with 20 years, and $34 \%$ (95\% CI: 33-34\%) with up to 39 years.

Conclusion: Most recurrent MIs were identified with a look-back period of 24 years. Including recurrent MIs in a planned incident MI cohort, due to shorter look-back periods, overestimated the mortality risk.

Keywords: acute myocardial infarction, bias, cardiology, cohort, epidemiology, methodology

\section{Introduction}

Acute myocardial infarction (MI) has a substantial impact on public health worldwide and causes more than one-third of deaths annually in developed countries. ${ }^{1}$ Survival after MI has improved in recent decades, ${ }^{2}$ yielding a growing number of individuals at risk of MI recurrence, ie, an MI occurring $>28$ days after a previous $\mathrm{MI}^{3}$ Compared with patients suffering from an incident MI, patients with recurrent MI are typically older and have a higher burden of comorbidities. Recurrent MIs are also associated with higher all-cause mortality. ${ }^{4}$

Accordingly, many studies seek to include patients only with incident MIs. To exclude patients with recurrent MI, studies based on historical data typically apply
Correspondence: Søren Korsgaard Department of Clinical Epidemiology, Aarhus University Hospital, Olof Palmes Allé 43-45, Aarhus N, Aarhus, 8200, Denmark

Tel +4587167212

Fax +4587167215

Email soren@clin.au.dk 
a "look-back" period, ie, searching the patient's hospital history for previous MI hospitalizations a given number of years back. If a previous MI hospitalization is found, the index event is defined as a recurrent MI. However, an optimal look-back period to accurately identify MI events as incident or recurrent is not well established. Moreover, left censoring poses a challenge. ${ }^{5}$ For instance, Medicare databases in the US and Techniker Krankenkasse databases in Germany contain data on patients only for a limited time period. In the Medicare databases, data collection typically begins at age $65 .^{6}$ Thus, it is unknown if a patient had been hospitalized with an MI earlier in life and impossible to determine whether a presumed first-time MI is actually an incident event. Accordingly, when lefttruncated registries are utilized, some recurrent MI cases will be included erroneously in a cohort planned to consist of incident cases.

The current study had three overall aims:

Aim 1: To examine the relation between the length of the look-back period and the proportion of MIs identified;

Aim 2: To examine how the relation between lookback period and identification of recurrent MIs (Aim 1) depends on age, sex, and presence of cardiovascular diseases; and

Aim 3: To examine the impact on survival estimates of including recurrent MIs in a cohort planned to consist only of incident MIs.

\section{Materials and Methods}

\section{Setting and Data Sources}

We conducted this study in Denmark, which had 5.7 million inhabitants in 2016. ${ }^{7}$ The Danish National Health Service provides tax-supported health care guaranteeing unfettered access to general and specialist medical practitioners, hospitals, and partial reimbursement for prescribed medications. ${ }^{8}$ All Danish residents are assigned a unique ten-digit Central Personal Register (CPR) number at birth or upon immigration, allowing unambiguous individual-level linkage of data from various Danish registries. ${ }^{9}$

We identified our study cohort through the Danish National Patient Registry. ${ }^{10}$ This Registry was established in 1977 and contains information on every non-psychiatric inpatient admission since 1978 and on every hospital outpatient clinic contact since $1995 .{ }^{10}$ Date of admission, date of discharge, urgency of admission (acute or elective), one primary diagnosis, and optionally several secondary diagnoses are recorded for each hospital contact. Diseases are coded according to the International Classification of Diseases (ICD). ${ }^{10}$ The Eighth Revision (ICD-8) was used until the end of 1993, and subsequently the Tenth Revision (ICD-10). ${ }^{10}$

Information on date of birth and vital status was obtained from the Danish Civil Registration System, which issues CPR numbers and records changes in vital status and migration for all Danish residents with daily electronic updates. ${ }^{9}$

We collected information on medication use from the Danish National Prescription Registry, which contains information on all prescription medications redeemed at Danish community pharmacies since $1995 .^{11}$ Medications are coded according to the Anatomical Therapeutic Chemical Classification (ATC) system.

\section{Index Myocardial Infarction Events}

We included all patients aged $\geq 18$ years with a hospital admission for MI from 1 January 2010 through 31 December 2016. We defined an MI as an inpatient hospital contact with MI recorded as a primary or secondary diagnosis in the Danish National Patient Registry. The accuracy of MI hospital-based diagnoses in the Danish National Patient Registry previously has been validated with a positive predictive value between $92 \%$ and $97 \% .^{10,12}$ The date of admission for an MI was defined as the "index date". In case of multiple MI admissions during 2010-2016, the first MI in the period was used as the index date. The first MI in the period 2010-2016 was considered the "index event".

\section{Cardiovascular Diseases}

We identified cardiovascular and other chronic diseases (ie, diabetes, hypertension, hypercholesterolemia, atrial fibrillation or flutter, ventricular tachyarrhythmias, heart failure, stroke, and kidney disease) based on Danish National Patient Registry data, using all available primary and secondary diagnoses from all recorded inpatient and outpatient hospital contacts. In addition to data from the Danish National Patient Registry, we extracted information on redeemed prescriptions for antidiabetic medications, antihypertensive medications, and statins from the Danish National Prescription Registry ${ }^{11}$ for the 5-year period preceding the index date. Medicationdefined hypertension consisted of at least two redeemed prescriptions within 180 days prior to the index date for medications in at least two different antihypertensive drug classes. ${ }^{13}$ The ICD and ATC codes used in the study are provided in Table S1. 


\section{Aim I: The Relation Between the Length of the Look-Back Period and the Proportion of Mls Identified}

While all index events occurred in patients with a first admission for MI during 2010-2016, some patients might have been admitted with an MI prior to 2010, ie, some index events could in fact be recurrent MIs. The first aim was to examine the proportion of index events that would be identified as recurrent MIs as a function of the lookback period - from a 1-year to the maximum 39-year lookback period. Moreover, we made a definition of a plateau as the number of look-back years required to identify $\leq 1 \%$ fewer index events as recurrent MIs, compared to applying the maximum look-back period (ie, up to 39 years).

\section{Aim 2: The Relation Between the Length of the Look-Back Period and the Proportion of Mls Identified Stratified by Age, Sex, and Cardiovascular Diseases}

The method described above for Aim 1 was repeated with stratification for the following age groups: $18-49$ years, 50-59 years, 60-69 years, $70-79$ years, and $\geq 80$ years. We further stratified on sex and presence of cardiovascular diseases.

\section{Aim 3: The Impact of the Look-Back Period on Survival Estimates}

The third aim was to explore whether including recurrent MIs in a planned incident cohort due to a short look-back period would impact survival estimates. Based on index events, we defined five different cohorts by applying different look-back periods to identify MI admissions prior to 2010. Application of the look-back periods resulted in classifying some index events as recurrent MIs to exclude from the intended incident cohort. We utilized look-back periods of 0 years, 5 years, 10 years, 20 years, and a maximum number of years (up to 39 years).

\section{Statistical Analysis}

The analyses performed on the five cohorts described under Aim 3 will be outlined in the following. We tabulated the distributions of age, sex, year of diagnosis, electrocardiogram-defined subtype of MI [non-ST-segment elevation MI (NSTEMI) and ST-segment elevation MI (STEMI)], diabetes, hypertension, hypercholesterolemia, atrial fibrillation or flutter, ventricular tachyarrhythmias, heart failure, stroke, and kidney disease.
We followed the patients from their index date until death from any cause, 5 years of follow-up, or the end of the study on 31 December 2016, whichever came first. We computed 5-year cumulative incidences and confidence intervals (CIs) of all-cause mortality using the 1-KaplanMeier method for the five cohorts. As a measure of overestimation of all-cause mortality risk stemming from including recurrent MIs, we calculated risk differences using the cohort defined by the maximum look-back period as the reference, eg, the risk difference between the cohort defined by 0 years of look-back compared with the cohort defined by maximum look-back.

To explore whether the relation between length of the look-back period and overestimation in 5-year all-cause mortality risk, we repeated the all-cause mortality analysis with stratification on the following age groups: 18-49 years, 5059 years, $60-69$ years, $70-79$ years, and $\geq 80$ years.

\section{Sensitivity Analysis}

Some of the index events identified as recurrent MIs might not represent a truly new event, but rather a second record of a previous diagnosis in a subsequent admission. To investigate this further, we stratified the Aim 1 analysis by diagnosis type (primary or secondary) and urgency of admission (acute or elective).

All statistical analyses were performed using Rstudio version 3.6.1 (R Foundation for Statistical Computing, Vienna, Austria). According to Danish law, ethical approval and informed consent were not required for this registry-based study. The study was reported to the Danish Data Protection Agency through institutional registration (record number 2014-54-0922, Aarhus University record number 2016-051-000001-811).

\section{Results}

We identified 65,210 patients hospitalized with an MI from 2010 through 2016 . We excluded 1,311 patients with missing information on age and sex and 14 patients aged $<18$ years at diagnosis, arriving at a total of 63,885 patients with index events.

\section{The Relation Between the Length of the Look-Back Period and the Proportion of Mls Identified}

Figure 1 shows the proportion of index events identified as recurrent MIs as a function of the number of look-back years. The proportion increased as the look-back period 


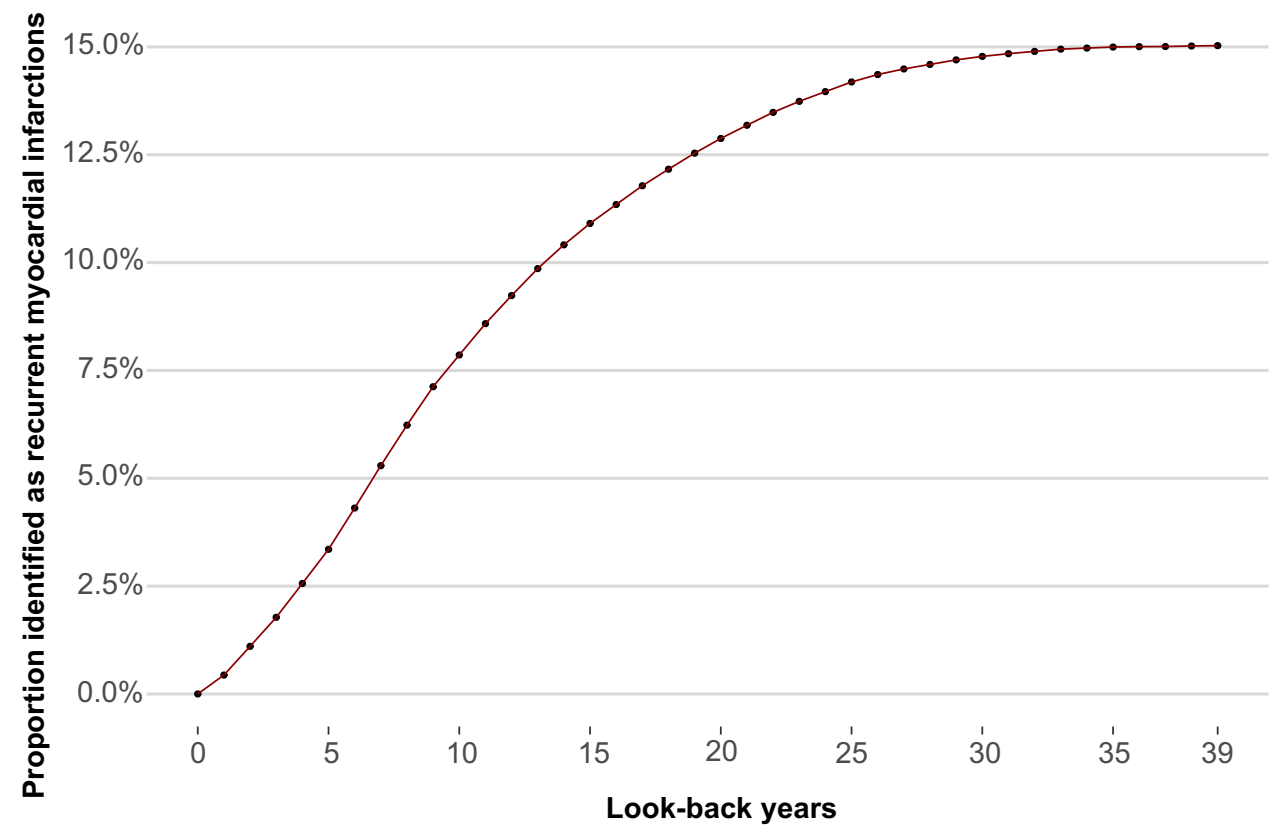

Figure I Proportion of index myocardial infarction events (2010-2016) identified as recurrent myocardial infarctions as a function of look-back years.

became longer. With the maximum look-back period (up to 39 years), $15 \%$ of index events were identified as recurrent MIs. A plateau was reached at 24 years of lookback.

\section{The Relation Between the Length of the Look-Back Period and the Proportion of Mls Identified Stratified by Age, Sex, and Cardiovascular Diseases}

Figure 2 shows the percentage of index events identified as recurrent MIs as a function of look-back years stratified by age groups. The figure demonstrates that 1) the percentage of recurrent MIs was 5.7\% among patients aged 18-49 years, $10 \%$ among patients aged 50-59 years, $13 \%$ among patients aged 60-69 years, 18\% among patients aged 70-79 years, and $20 \%$ among patients aged $\geq 80$ years; 2 ) the number of look-back years required to reach a plateau differed across age groups, with 10 years for the 18-49-year age group, 15 years for the 50-59-year age group, 21 years for the 60-69year age group, 25 years for the 70-79-year age group, and 27 years for the $\geq 80$-year age group.

Sex-stratified analyses showed that the proportion of index events identified as recurrent MIs was higher for males, but the relation between proportion identified and look-back years was nearly the same between sexes (Figure S1).
The proportions of index events identified as recurrent MIs with the maximum look-back period (up to 39 years) and time to plateau, stratified by the presence of cardiovascular diseases, are provided in Table 1. For all cardiovascular diseases, the proportion of index events identified as recurrent MIs using the maximum look-back period was higher compared to index events in patients without a history of the diseases. The highest proportions of index events found to be recurrent MIs were identified among patients with a history of ventricular tachyarrhythmia and heart failure. However, for all cardiovascular diseases, time to plateau differed little between index events among patients with and without a cardiovascular disease history.

\section{The Impact of the Look-Back Period on Survival Estimates}

Table 2 presents patient characteristics for five different "incident" cohorts of MIs defined by 0 years, 5 years, 10 years, 20 years, and up to 39 years of look-back. Compared to a short or no look-back period, a maximum look-back period (up to 39 years) yielded a higher proportion of patients with a history of cardiovascular diseases, eg, hypertension (47\% vs 43\%), hypercholesterolemia (33\% vs 26\%), heart failure (12\% vs $7.8 \%$ ), and stroke (11\% vs $10 \%)$.

Figure 3 shows risk estimates of cumulative all-cause mortality plotted for the five different cohorts. The 5-year risk of all-cause mortality was 36\% (95\% CI: 36-37\%) for 


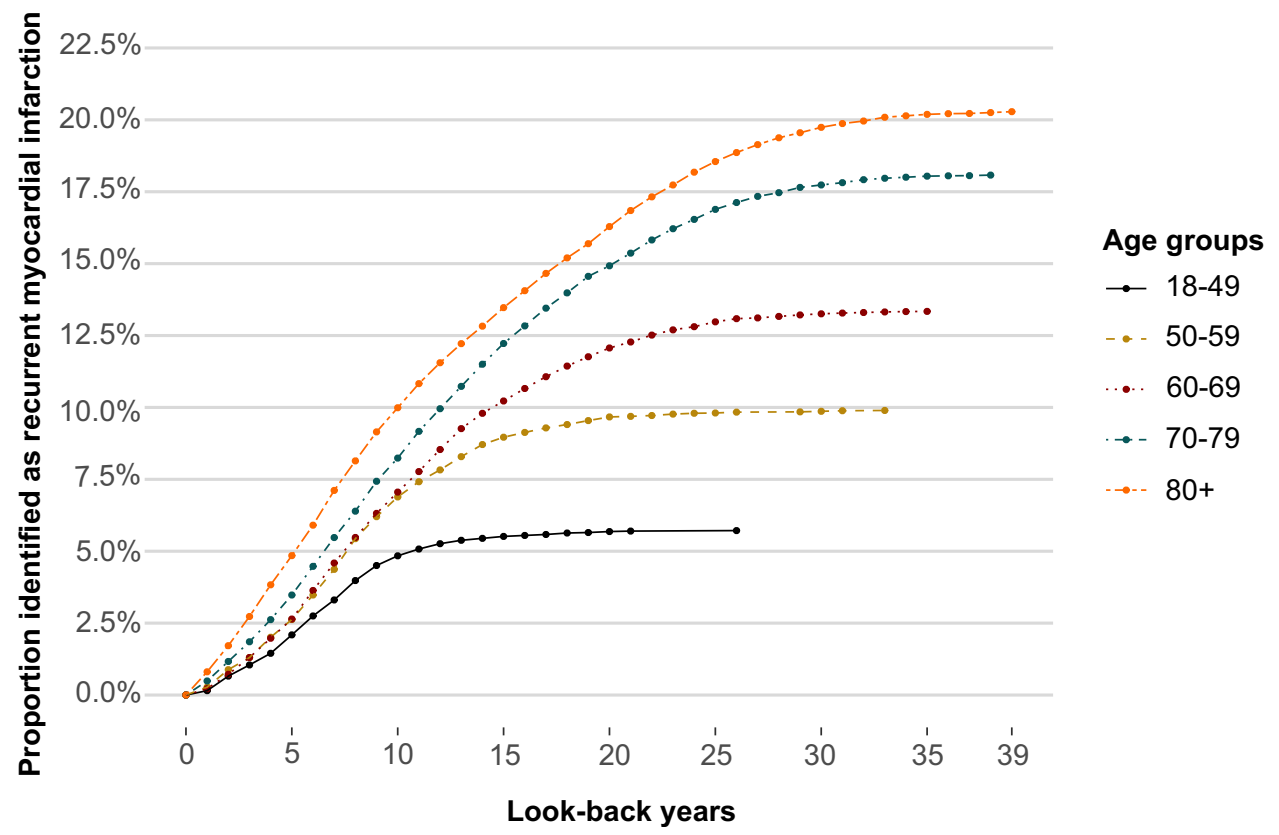

Figure 2 Proportion of index myocardial infarction events (2010-2016) identified as recurrent myocardial infarctions as a function of look-back years. Stratified by age groups.

the cohort defined by 0 years of look-back, 35\% (95\% CI: $35-36 \%$ ) for the cohort defined by 5 years of look-back, $35 \%$ (95\% CI: 35-36\%) for the cohort defined by 10 years of lookback, 34\% (95\% CI: 34-35\%) for the cohort defined by 20 years of look-back, and 34\% (95\% CI: 33-34\%) for the cohort defined by the maximum look-back period (up to 39 years). Thus, applying 0 years of look-back resulted in a $2.8 \%$ (95\% CI: $2.2-3.5 \%)$ overestimation of 5-year allcause mortality risk. For 5 years of look-back, the overestimation was $2.0 \%$ (95\% CI: $1.3-2.7 \%)$. For 10 years of lookback, the overestimation was 1.5\% (95\% CI: $0.8-2.2 \%)$, and for 20 years of look-back, the overestimation was $0.6 \%(95 \%$ CI: $-0.1-1.3 \%)$.

Across all age groups, we found an overestimation in 5-year all-cause mortality risk with shorter look-back periods, but the overestimation was highest among patients aged $\geq 50$ years, and particularly for those aged between 70 and 79 years (Table S2).

\section{Sensitivity Analysis}

When the index event was coded as a secondary diagnosis, the proportion identified as recurrent MIs with the maximum look-back period (up to 39 years) was larger than the proportion identified as recurrent MIs when the index event was coded as a primary diagnosis (20\% vs $14 \%$ ). The time to plateau was 25 years when the index event was coded as a secondary diagnosis, and 24 years when the index event was coded as a primary diagnosis. Similarly, $18 \%$ of index events were identified as recurrent MIs when the hospital admission was elective compared with $15 \%$ when the admission was acute. The time to plateau was 24 years for both acute and elective admissions (Table 1).

\section{Discussion \\ Key Results}

When using the maximum look-back period (up to 39 years), $15 \%$ of index events were found to be recurrent MIs. A plateau in the relation between the length of lookback period and the proportion of index events identified as recurrent MI was reached with 24 years of look-back. The proportion of index events identified as recurrent MIs and the time required to reach a plateau increased with age. A history of any individual cardiovascular disease was associated with a higher proportion of index events being identified as recurrent MIs, but there was little difference in the time to plateau. Inclusion of recurrent MIs in an intended incident MI study cohort overestimated the absolute risk of 5-year all-cause mortality. The overestimation decreased with increasing length of the lookback period. 
Table I Proportion of Index Events Identified as Recurrent Myocardial Infarctions with the Maximum Look-Back Period, and Time to Plateau by History of Cardiovascular Diseases and Diagnostic Characteristics of the Index Event.

\begin{tabular}{|c|c|c|}
\hline & $\begin{array}{l}\text { Identified as } \\
\text { Recurrent } \\
\text { Myocardial } \\
\text { Infarctions }\end{array}$ & $\begin{array}{c}\text { Time } \\
\text { to Plateau }\end{array}$ \\
\hline & (\%) & (Years) \\
\hline \multicolumn{3}{|c|}{ Cardiovascular comorbidities } \\
\hline Atrial fibrillation/flutter & 26 & 26 \\
\hline No & 14 & 24 \\
\hline Ventricular tachyarrhythmia & 53 & 31 \\
\hline No & 15 & 33 \\
\hline Hypercholesterolemia & 33 & 26 \\
\hline No & 6.6 & 22 \\
\hline Hypertension & 24 & 26 \\
\hline No & 7.2 & 21 \\
\hline Diabetes & 24 & 25 \\
\hline No & 13 & 24 \\
\hline Heart failure & 42 & 27 \\
\hline No & 12 & 23 \\
\hline Stroke & 23 & 27 \\
\hline No & 14 & 24 \\
\hline Kidney disease & 26 & 25 \\
\hline No & 14 & 24 \\
\hline \multicolumn{3}{|c|}{ Characteristics of diagnosis } \\
\hline \multicolumn{3}{|l|}{ Diagnosis type } \\
\hline Primary & 14 & 24 \\
\hline Secondary & 20 & 25 \\
\hline \multicolumn{3}{|l|}{ Urgency of admission } \\
\hline Acute & 15 & 24 \\
\hline Elective & 18 & 24 \\
\hline
\end{tabular}

\section{Limitations}

Some limitations must be considered when interpreting our results. When an MI is suspected in Denmark, patients are immediately transferred to a public hospital and the admission is recorded in the Danish National Patient Registry. Inpatient data from this source should yield high completeness of true MI occurrences. Despite our minimum lookback period of 33 years, some data still might be missing on previous MI hospitalizations. However, since the proportion of index events identified as recurrent MIs plateaued, we probably detected the vast majority of previous MI hospitalizations. However, it is likely that even with a long look-back period, some events classified as incident
MIs are in fact not incident events as patients might have suffered from a previous unrecognized MI. ${ }^{14}$

The impact on our findings of a second record of the same MI is difficult to gauge, as we had no way of ascertaining with certainty a truly new event from a second record of an earlier event. In our sensitivity analysis, we found that a higher proportion of index events in fact were recurrent MIs (with examination of all available hospital history) when the index event was recorded as a secondary diagnosis or as an elective admission, compared to a primary diagnosis or acute admission. Speculating that secondary diagnoses and elective admissions are associated with second records of a given event, some of the index events we classified as recurrent MIs could be such second records.

The validity of diagnoses of the cardiovascular comorbidities in the Danish National Patient Registry has been evaluated previously, and the positive predictive values are as follows: hypertension (92\%), hypercholesterolemia (96\%), atrial fibrillation or flutter $(95 \%)$, ventricular tachyarrhythmias $(80 \%)$, heart failure $(76 \%)$, stroke (94\%), and kidney disease (100\%). ${ }^{12,15}$ Using data from the Danish National Patient Registry and data on prescription medications to identify a history of diabetes has previously been evaluated and found to have a positive predictive value of $97 \%{ }^{16}$

For the survival analysis, we used information on vital status from the Central Personal Register. These data are highly valid and virtually complete. ${ }^{9}$

\section{Comparison to Other Studies}

Sulo et al examined whether the length of the look-back period had any impact on trends in MI incidence and mortality for a Norwegian cohort of index events occurring during 2004-2009. ${ }^{17}$ Moreover, they computed a rate ratio of the incidence rates in 2004 and 2009. They applied look-back periods of $3,5,7,8$, and 10 years. The investigators concluded that applying a look-back period shorter than 10 years led to overestimation of the absolute number of incident events. However, only look-back periods shorter than 7 years produced incidence rate ratios that differed from results of analyses with a 10-year look-back period. They found an overestimation in 1-year all-cause mortality when using a look-back period of 3 years compared with a look-back period of 10 years. The overestimation in all-cause mortality was particularly present among individuals aged $\geq 65$ years. The present study 
Table 2 Patient Characteristics for Incident Myocardial Infarction Cohorts Defined by Different Look-Back Periods, Denmark, 20I02016

\begin{tabular}{|c|c|c|c|c|c|}
\hline & \multicolumn{5}{|c|}{ Look-Back Period } \\
\hline & 0 Years & 5 Years & 10 Years & 20 Years & Up to 39 Years \\
\hline & n (\%) & n (\%) & n (\%) & n (\%) & n (\%) \\
\hline Total (1 00\%) & 63,885 & 61,743 & 58,865 & 55,659 & 54,284 \\
\hline Age (median, QI-Q3) & $70(60-80)$ & $70(60-80)$ & $70(60-80)$ & $70(60-80)$ & 69 (59-79) \\
\hline Sex, male & $4 I, 224(65 \%)$ & $39,840(65 \%)$ & $37,805(64 \%)$ & $35,439(64 \%)$ & 34,391 (63\%) \\
\hline \multicolumn{6}{|l|}{ Calendar period } \\
\hline 2010 & $10,509(16 \%)$ & 9445 (15\%) & $8950(15 \%)$ & 8479 (I5\%) & 8290 (15\%) \\
\hline 2011 & $9556(15 \%)$ & $9012(15 \%)$ & $8553(15 \%)$ & 8095 (I5\%) & 7888 (15\%) \\
\hline 2012 & $8953(14 \%)$ & 8641 (14\%) & 8199 (14\%) & 7767 (I4\%) & $7586(14 \%)$ \\
\hline 2013 & $8735(14 \%)$ & $8572(14 \%)$ & $8126(14 \%)$ & 7687 (14\%) & $748 \mid(14 \%)$ \\
\hline 2014 & $9083(14 \%)$ & 9024 (15\%) & 8577 (15\%) & $8060(15 \%)$ & 7864 (I5\%) \\
\hline 2015 & $8810(14 \%)$ & $8810(14 \%)$ & $8462(14 \%)$ & 8011 (14\%) & 7785 (14\%) \\
\hline 2016 & $8239(13 \%)$ & 8239 (13\%) & 7998 (14\%) & $7560(14 \%)$ & 7390 (14\%) \\
\hline \multicolumn{6}{|l|}{ Subtype of MI } \\
\hline NSTEMI & $29,277(46 \%)$ & $28,199(46 \%)$ & $26,688(45 \%)$ & $24,969(45 \%)$ & $24,178(45 \%)$ \\
\hline STEMI & I5,398 (24\%) & $15,130(25 \%)$ & $14,636(25 \%)$ & $14,189(26 \%)$ & $14,048(26 \%)$ \\
\hline Unclassified & $19,210(30 \%)$ & $|8,4| 4(30 \%)$ & $|7,54|(30 \%)$ & $16,50 \mid(30 \%)$ & $16,058(30 \%)$ \\
\hline \multicolumn{6}{|l|}{ Cardiovascular diseases } \\
\hline Diabetes & 12,048 (19\%) & $11,416(19 \%)$ & $10,538(18 \%)$ & 9547 (17\%) & 9185 (17\%) \\
\hline Hypertension & $30,217(47 \%)$ & $28,504(46 \%)$ & 26,347 (45\%) & $24,004(43 \%)$ & $23,047(43 \%)$ \\
\hline Hypercholesterolemia & $20,769(33 \%)$ & $19,190(31 \%)$ & I7,063 (29\%) & $|4,8| 7(27 \%)$ & $13,999(26 \%)$ \\
\hline Atrial fibrillation or flutter & 7394 (I2\%) & $6914(11 \%)$ & $6364(11 \%)$ & $5744(10 \%)$ & $5456(10 \%)$ \\
\hline Ventricular tachyarrhythmia & $858(1.3 \%)$ & $780(1.3 \%)$ & $651(1.1 \%)$ & $503(0.9 \%)$ & $406(0.7 \%)$ \\
\hline Heart failure & $7315(12 \%)$ & 6458 (II\%) & $5574(10 \%)$ & 4605 (8.3\%) & $4211(7.8 \%)$ \\
\hline Stroke & $6790(11 \%)$ & $6417(10 \%)$ & $5975(10 \%)$ & $5488(10 \%)$ & $5241(10 \%)$ \\
\hline Kidney disease & 3722 (5.8\%) & $3462(5.6 \%)$ & 3187 (5.4\%) & $288 \mathrm{I}(5.2 \%)$ & $2762(5.1 \%)$ \\
\hline
\end{tabular}

Abbreviations: MI, myocardial infarction; NSTEMI, non-ST-segment elevation myocardial infarction; STEMI, ST-segment elevation myocardial infarction.

extends this Norwegian study by providing information on the relation between look-back period and proportion of index events identified as recurrent MIs. Furthermore, we were able to apply a longer maximum look-back period, revealing that even 10 years of look-back was insufficient to detect all previous MIs. Moreover, our study supports that the length of the look-back period has an impact on all-cause mortality, when the period allows for long follow-up.

\section{Interpretation}

Rather than altering clinical management, the aim with the present study was to provide methodological insights useful for researchers using incident MI cohorts. The results from this study can assist in judiciously choosing the length of look-back periods, when designing studies of MI focusing on incident events.

The age-stratified analyses further permit more specific decision-making based on the age distribution in the population under study. Investigating an elderly population requires data allowing a longer look-back period, compared to a study of a younger population. This is consistent with the higher probability of a previous MI among older patients.

The proportion of index events identified as recurrent MIs when the maximum look-back period was applied was higher for the index patients with a history of cardiovascular diseases. This might be 1) due to older age among the patients with index events who had truly recurrent MIs; 2) due to a first MI contributing either independently 


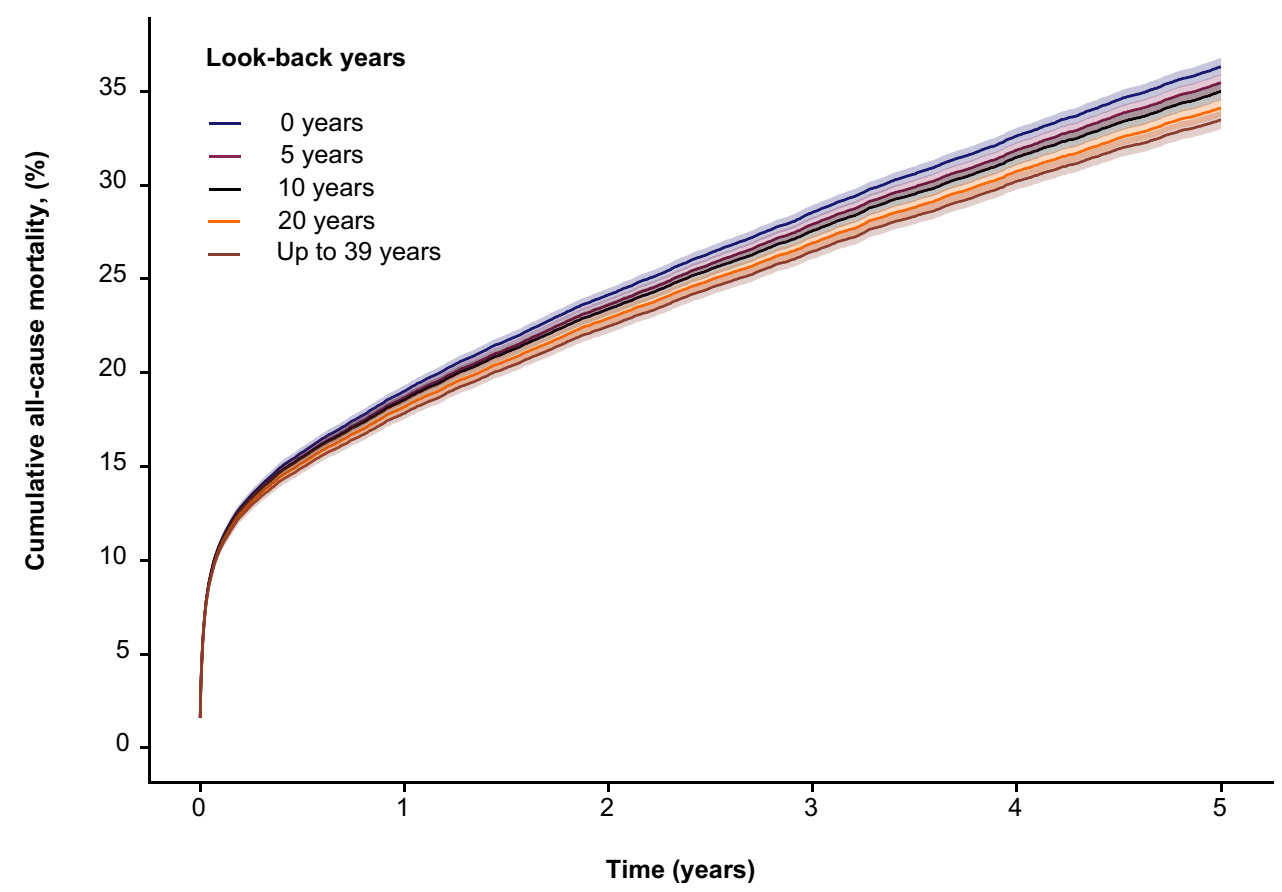

Figure 3 Five-year cumulative incidence of all-cause mortality for five different cohorts of myocardial infarction defined by $0,5,10,20$, and up 39 years of look-back.

or through shared risk factors to the development of comorbidities, eg, heart failure, ${ }^{18}$ atrial fibrillation, ${ }^{19-21}$ stroke, ${ }^{22}$ and kidney disease, ${ }^{23}$ or 3 ) due to the examination of patients suffering from an MI for well-established risk factors, such as hypertension, hypercholesterolemia, diabetes, and kidney disease. ${ }^{24}$ Such a work-up increases the likelihood of having these diseases recognized and subsequently recorded in the Danish National Patient Registry at the time of a subsequent hospitalization for MI. However, it is noteworthy that the look-back time before reaching a plateau in the proportion of index events identified as recurrent MIs did not differ greatly between patients with and without a history of cardiovascular diseases. It therefore seems that the length of a look-back period can be chosen somewhat independently of these cardiovascular comorbidities. Although the time to a plateau was generally a few years longer among index MI patients having cardiovascular comorbidities.

If the length of available data on hospital history prevents a long look-back period, results from the present study might be pertinent in discussing the validity of a study cohort intended to consist of incident MIs. Similarly, our results quantify potential overestimation of all-cause mortality stemming from applying short lookback periods.
It has been questioned whether data based on ICD-8 diagnoses should continue to be used. Our findings argue that all available data ought to be considered in choosing look-back periods.

\section{Conclusion}

Applying a look-back period of 24 years identified most of the index events that were recurrent MIs and therefore should be excluded from the incident MI cohort. By applying shorter look-back periods, and consequently including recurrent MIs into a planned incident MI cohort, risk estimates of 5-year all-cause mortality were overestimated.

\section{Funding}

This project was supported by the Independent Research Fund Denmark (grant 9149-00025B). The funding organization had no role in the design and conduct of the study, collection and analysis of the data, or reporting of the study.

\section{Disclosure}

Department of Clinical Epidemiology is involved in studies with funding from various companies as research grants to (and administered by) Aarhus University, but 
none of these studies have any relation to the submitted work. Dr Schmidt receives grant support from the Novo Nordisk Foundation (grant NNF19OC0054908) for research unrelated to the submitted work. Mr Søren Korsgaard reports grants from the Independent Research Fund Denmark, during the conduct of the study. The authors report no other conflicts.

\section{References}

1. Reed GW, Rossi JE, Cannon CP. Acute myocardial infarction. Lancet. 2017;389(10065):197-210. doi:10.1016/S0140-6736(16)30677-8

2. Schmidt M, Jacobsen JB, Lash TL, Bøtker HE, Sørensen HT. 25 year trends in first time hospitalisation for acute myocardial infarction, subsequent short and long term mortality, and the prognostic impact of sex and comorbidity: a Danish nationwide cohort study. BMJ. 2012;344:e356. doi:10.1136/bmj.e356

3. Thygesen K, Alpert JS, Jaffe AS, et al. Fourth universal definition of myocardial infarction (2018). J Am Coll Cardiol. 2018;72 (18):2231-2264. doi:10.1016/j.jacc.2018.08.1038

4. Gerber Y, Weston SA, Jiang R, Roger VL. The changing epidemiology of myocardial infarction in Olmsted County, Minnesota, 1995-2012. Am J Med. 2015;128(2):144-151. doi:10.1016/j.amjmed.2014.09.012

5. Toft sørensen H, Barron J. Registries and medical databases. In: Olsen J, Saracci R, Trichopoulos D, editors. Teaching Epidemiology: A Guide for Teachers in Epidemiology, Public Health and Clinical Medicine. 3rd. Oxford University Press; 2010.

6. Mues KE, Liede A, Liu J, et al. Use of the medicare database in epidemiologic and health services research: a valuable source of real-world evidence on the older and disabled populations in the US. Clin Epidemiol. 2017;9:267-277. doi:10.2147/CLEP.S105613

7. Statistics Denmark. Denmark in figures; 2016. Available from: https://www.dst.dk/da/Statistik/Publikationer/VisPub?cid=21498. Accessed July 20, 2020.

8. Schmidt M, Schmidt SAJ, Adelborg K, et al. The Danish health care system and epidemiological research: from health care contacts to database records. Clin Epidemiol. 2019;11:563-591. doi:10.2147/ CLEP.S179083

9. Schmidt M, Pedersen L, Sørensen HT. The Danish Civil Registration System as a tool in epidemiology. Eur J Epidemiol. 2014;29 (8):541-549. doi:10.1007/s10654-014-9930-3

10. Schmidt M, Schmidt SA, Sandegaard JL, Ehrenstein V, Pedersen L, Sorensen HT. The Danish National Patient Registry: a review of content, data quality, and research potential. Clin Epidemiol. 2015;7:449-490. doi:10.2147/CLEP.S91125

11. Pottegård A, Schmidt SAJ, Wallach-Kildemoes H, Sørensen HT, Hallas J, Schmidt M. Data resource profile: the Danish National Prescription Registry. Int J Epidemiol. 2017;46(3):798-798f.
12. Sundboll J, Adelborg K, Munch T, et al. Positive predictive value of cardiovascular diagnoses in the Danish National Patient Registry: a validation study. BMJ Open. 2016;6(11):e012832. doi:10.1136/ bmjopen-2016-012832

13. Olesen JB, Lip GY, Hansen ML, et al. Validation of risk stratification schemes for predicting stroke and thromboembolism in patients with atrial fibrillation: nationwide cohort study. BMJ. 2011;342:d124. doi:10.1136/bmj.d124

14. Dörr M. Silent myocardial infarction: the risk beyond the first admission. Heart. 2010;96(18):1434-1435. doi:10.1136/hrt.2010.201384

15. Thygesen SK, Christiansen CF, Christensen S, Lash TL, Sørensen HT. The predictive value of ICD-10 diagnostic coding used to assess Charlson comorbidity index conditions in the population-based Danish National Registry of Patients. BMC Med Res Methodol. 2011;11:83. doi:10.1186/1471-2288-11-83

16. Thomsen RW, Hundborg HH, Lervang HH, Johnsen SP, Sørensen HT, Schønheyder HC. Diabetes and outcome of community-acquired pneumococcal bacteremia: a 10-year population-based cohort study. Diabetes Care. 2004;27(1):70-76. doi:10.2337/diacare.27.1.70

17. Sulo G, Igland J, Vollset SE, et al. Effect of the lookback period's length used to identify incident acute myocardial infarction on the observed trends on incidence rates and survival: cardiovascular disease in Norway project. Circ Cardiovasc Qual Outcomes. 2015;8 (4):376-382. doi:10.1161/CIRCOUTCOMES.114.001703

18. Chamberlain AM, Boyd CM, Manemann SM, et al. Risk factors for heart failure in the community: differences by age and ejection fraction. Am $J$ Med. 2020;133(6):e237-e248. doi:10.1016/j. amjmed.2019.10.030

19. Brandes A, Smit MD, Nguyen BO, Rienstra M, Van Gelder IC. Risk factor management in atrial fibrillation. Arrhythm Electrophysiol Rev. 2018;7(2):118-127. doi:10.15420/aer.2018.18.2

20. Lip GYH, Coca A, Kahan T, et al. Hypertension and cardiac arrhythmias: executive summary of a consensus document from the European Heart Rhythm Association (EHRA) and ESC Council on Hypertension, endorsed by the Heart Rhythm Society (HRS), Asia-Pacific Heart Rhythm Society (APHRS), and Sociedad Latinoamericana de Estimulación Cardíaca y Electrofisiología (SOLEACE). Eur Heart J Cardiovasc Pharmacother. 2017;3(4):235-250.

21. Pallisgaard JL, Schjerning AM, Lindhardt TB, et al. Risk of atrial fibrillation in diabetes mellitus: a nationwide cohort study. Eur J Prev Cardiol. 2016;23(6):621-627. doi:10.1177/2047487315599892

22. Boehme AK, Esenwa C, Elkind MS. Stroke risk factors, genetics, and prevention. Circ Res. 2017;120(3):472-495. doi:10.1161/ CIRCRESAHA. 116.308398

23. McClellan WM. Epidemiology and risk factors for chronic kidney disease. Med Clin North Am. 2005;89(3):419-445. doi:10.1016/j. mona.2004.11.006

24. Anand SS, Islam S, Rosengren A, et al. Risk factors for myocardial infarction in women and men: insights from the INTERHEART study. Eur Heart J. 2008;29(7):932-940. doi:10.1093/eurheartj/ehn018
Clinical Epidemiology

\section{Publish your work in this journal}

Clinical Epidemiology is an international, peer-reviewed, open access, online journal focusing on disease and drug epidemiology, identification of risk factors and screening procedures to develop optimal preventative initiatives and programs. Specific topics include: diagnosis, prognosis, treatment, screening, prevention, risk factor modification,

Submit your manuscript here: https://www.dovepress.com/clinical-epidemiology-journal systematic reviews, risk \& safety of medical interventions, epidemiology \& biostatistical methods, and evaluation of guidelines, translational medicine, health policies \& economic evaluations. The manuscript management system is completely online and includes a very quick and fair peer-review system, which is all easy to use. 